\title{
Learning Tomography Assessed Using Mie Theory
}

\author{
Joowon Lim, ${ }^{1, *}$ Alexandre Goy, ${ }^{1}$ Morteza H. Shoreh, ${ }^{1}$ Michael Unser, ${ }^{2}$ and Demetri Psaltis ${ }^{1}$ \\ ${ }^{1}$ Optics Laboratory, École Polytechnique Fédérale de Lausanne (EPFL), 1015 Lausanne, Switzerland \\ ${ }^{2}$ Biomedical Imaging Group, École Polytechnique Fédérale de Lausanne (EPFL),
} 1015 Lausanne, Switzerland

(Received 20 June 2017; revised manuscript received 24 November 2017; published 27 March 2018)

\begin{abstract}
In optical diffraction tomography, the multiply scattered field is a nonlinear function of the refractive index (RI) of the object. The Rytov method relies on a single-scattering propagation model and is commonly used to reconstruct images. Recently, a reconstruction model was introduced based on the beam propagation method that takes multiple scattering into account. We refer to this method as learning tomography (LT). We carry out simulations and experiments in order to assess the performance of LT over the iterative single-scattering propagation method. Each algorithm is rigorously assessed for spherical and cylinderical objects, with synthetic data generated using Mie theory. By varying the RI contrast and the size of the objects, we show that the LT reconstruction is more accurate and robust than the reconstruction based on the single-scattering propagation model. In addition, we show that LT is able to correct distortions that are evident in the Rytov-approximation-based reconstructions due to limitations in phase unwrapping. More importantly, the ability of LT to handle multiple scattering is demonstrated by simulations of multiple cylinders using Mie theory and is confirmed by experiment.
\end{abstract}

DOI: 10.1103/PhysRevApplied.9.034027

\section{INTRODUCTION}

Quantitative-phase-imaging (QPI) microscopy measures sample-induced phase delay, which relates to the refractive index (RI) contrast and the sample thickness. Each material has its own distinct RI value, and QPI can therefore provide physiological information [1] such as the structure and dynamics of cells [2,3], the quantification of specific molecules [4,5], and the dry mass [6,7]. Optical diffraction tomography (ODT) is a QPI method that enables us to visualize 3D RI distributions from multiple 2D scattered fields acquired at various illumination angles [8-10]. It provides the physiological information by measuring the 3D RI distribution without any exogenous labeling agents, making it a powerful tool for various physiological studies.

One limitation of ODT comes from the fact that views far from the optical axis are usually not accessible due to the limited numerical aperture of the optics. This is referred to as the missing cone problem. It generally causes an underestimation of the RI values and an elongation of RI tomograms along the optical axis [11,12]. The missing cone problem can be mediated using sparsity-based

*joowon.lim@epfl.ch

Published by the American Physical Society under the terms of the Creative Commons Attribution 4.0 International license. Further distribution of this work must maintain attribution to the author(s) and the published article's title, journal citation, and DOI. regularization algorithms [11,13]. A more fundamental problem is the inherent nonlinearity in scattering through inhomogeneous media [14]. These nonlinear effects are assumed to be negligible in conventional linear ODT frameworks (Born and Rytov approximations). Even though the approximations could be valid for a week scatterer such as a single cell [15], the validity of the approximations [16,17] restricts the application of ODT in complex samples. Distortions caused by multiple scattering can be especially severe for thick or high-contrast samples when several scattering objects are aggregated [18].

Reconstruction algorithms that consider the nonlinear process have recently been proposed [19-21]. We focus on the beam propagation method (BPM), which can be used as the propagation model combined with sparsity-based regularization in the iterative reconstruction scheme [20]. The BPM consists of a sequence of two substeps: diffraction followed by refraction [22]. The BPM can implement multiple scattering at different depths within the medium. Therefore, iterative reconstruction algorithms that combine the BPM and a sparsity-based regularization can outperform the conventional single-scattering model (Rytov approximation) [20,23].

The same reconstruction framework should be used, except for the propagation model, in order to fairly investigate the performance of the multiple-scattering model compared to that of the single-scattering model. In order to differentiate the performance of learning tomography (LT) from the effects of sparsity-based regularization, it should be 
compared with single-scattering tomography, which utilizes the same reconstruction algorithm scheme including sparsity-based regularization. In addition, in order to quantitatively compare any improvements made by the multiplescattering propagation model, we use Mie theory to provide the ground truth of the 3D RI distribution of the object.

In this paper, exactly the same algorithmic scheme $[20,23,24]$ is used for the single-scattering and multiplescattering algorithms except for the propagation model part. Simulated measurements are generated using Mie theory, whose analytical solution serves as the ground truth [25]. The two propagation models (single scattering and multiple scattering) are tested under three different schemes: (1) variable RI contrast with fixed size, (2) variable size with fixed RI contrast, and (3) different RI contrast and size with fixed sample-induced phase delay. In addition, we compare the ability of each model to deal with multiple scattering caused by multiple objects by testing the two models using the Mie theory for multiple cylinders [26]. After that, the performance of each model when phase unwrapping fails is discussed. Finally, we apply the algorithms to various experimental data and compare the experimental data to the simulation results. In order to obtain a direct comparison between the experiment and a computer simulation, we conduct experiments on optical fibers which can be considered cylinders. We conduct experiments on two fibers for three different configurations, and also for simulations using Mie theory for multiple cylinders to make a direct comparison between the single-scattering and multiple-scattering models. Experimental data are obtained for a sample consisting of two beads to confirm the capacity of LT to successfully handle multiple scattering. A more important issue is what the Mie theory results obtained for cylinders and spheres tell us about how well we can image objects such as cells. There has been prior research on applying Mie theory to spheroidal scatterers such as cell nuclei in biological materials [27]. We can think of biological cells as a rough aggregation of multiple spheres or cylinders. We carry out an experiment in which we image two cells arranged such that the light scattered from one of them illuminates the other. We observe clear tomographic reconstructions for the two-cell sample from LT, which we could not get using the single-scattering propagation model.

\section{THEORY}

\section{A. Optical diffraction tomography}

The Helmholtz equation describing scattering in an inhomogeneous medium can be written as

$$
\nabla^{2} U_{s}(\mathbf{r})+k^{2} U_{s}(\mathbf{r})=-4 \pi F(\mathbf{r}) U(\mathbf{r}),
$$

where $U(\mathbf{r})$ is the total electric field: the sum of the incident field $U_{i}(\mathbf{r})$ and the scattered field $U_{s}(\mathbf{r}) . F(\mathbf{r})=\left[k^{2} /(4 \pi)\right]$ $\left\{\left[n(\mathbf{r})^{2} /\left(n_{0}^{2}\right)\right]-1\right\}$ is the scattering potential of a sample with refractive index $n(\mathbf{r})$ immersed in a medium with refractive index $n_{0}$. The optical wavelength in free space is $\lambda$, resulting in the wave number $k=\left[\left(2 \pi n_{0}\right) / \lambda\right]$. The integral solution of Eq. (1) can be obtained using the homogeneous Green's function resulting in

$$
U_{s}(\mathbf{r})=\int_{V} F\left(\mathbf{r}^{\prime}\right) U\left(\mathbf{r}^{\prime}\right) G\left(\mathbf{r}-\mathbf{r}^{\prime}\right) d \mathbf{r}^{\prime},
$$

where $G\left(\mathbf{r}-\mathbf{r}^{\prime}\right)=\left[\left(e^{i k\left|\mathbf{r}-\mathbf{r}^{\prime}\right|}\right) /\left|\mathbf{r}-\mathbf{r}^{\prime}\right|\right]$ is the Green's function of the 3D Helmholtz equation, Eq. (1).

\section{B. Single-scattering propagation model}

The scattered field $U_{s}(\mathbf{r})$ in Eq. (2) is linear in $U_{i}(\mathbf{r})$ but nonlinear in $F(\mathbf{r})$. We can linearize the problem by replacing the total field, $U(\mathbf{r})$, with the incident field, $U_{i}(\mathbf{r})[14]:$

$$
U_{s}(\mathbf{r})=\int_{V} F\left(\mathbf{r}^{\prime}\right) U_{i}\left(\mathbf{r}^{\prime}\right) G\left(\mathbf{r}-\mathbf{r}^{\prime}\right) d \mathbf{r}^{\prime},
$$

which is the Born approximation. We can expand it to the Rytov approximation by simply changing the left term of Eq. (3) to $U_{i}(\mathbf{r}) \log \left\{[U(\mathbf{r})] /\left[U_{i}(\mathbf{r})\right]\right\}$, which can be thought of as the following first-order Taylor expansion of the scattered field: $U_{s}(\mathbf{r})=U_{i}(\mathbf{r})\{[U(\mathbf{r})] /$ $\left.\left[U_{i}(\mathbf{r})\right]-1\right\}=U_{i}(\mathbf{r})\left(e^{\log \left\{[U(\mathbf{r})] /\left[U_{i}(\mathbf{r})\right]\right\}}-1\right) \simeq U_{i}(\mathbf{r}) \log$ $\left\{[U(\mathbf{r})] /\left[U_{i}(\mathbf{r})\right]\right\}$. The two approximations share the same propagation model, which was originally derived from the Born approximation. Therefore, we refer to the right term of Eq. (3) as the single-scattering propagation model. However, these approximations (Born and Rytov) are only valid under the assumption of weak scattering. Necessary conditions of the approximations were well studied in Ref. [16] as follows:

$$
\begin{gathered}
\Delta n(\mathbf{r}) L<\frac{\lambda}{4} \text { (Born approximation), } \\
\Delta n(\mathbf{r}) \gg\left(\frac{\nabla \phi_{s} \lambda}{2 \pi}\right)^{2} \text { (Rytov approximation), }
\end{gathered}
$$

where $\Delta n(\mathbf{r})=n(\mathbf{r})-n_{0}, L$ is the radius of an object, and $\phi_{s}(\mathbf{r})=\log \left\{[U(\mathbf{r})] /\left[U_{i}(\mathbf{r})\right]\right\}$.

Under the assumption of plane-wave incidence, $U_{i}(\mathbf{r})=$ $e^{i \mathbf{k}^{\text {in }} \cdot \mathbf{r}}$, Eq. (3) can be transformed as follows [14]:

$$
\begin{aligned}
& \frac{k_{z}}{2 \pi i} \iint_{-\infty}^{+\infty} U_{s}(\mathbf{r} ; z=0) e^{-i\left(k_{x} x+k_{y} y\right)} d x d y \\
& \quad=\int_{V} F\left(\mathbf{r}^{\prime}\right) e^{-i\left(\mathbf{k}-\mathbf{k}^{\mathrm{in}}\right) \cdot \mathbf{r}^{\prime}} d \mathbf{r}^{\prime},
\end{aligned}
$$

where $U_{s}(\mathbf{r} ; z=0)$ is the measurement in the image plane and $k_{z}=\sqrt{k^{2}-k_{x}^{2}-k_{y}^{2}}$. Equation (6) is a linear 
relationship between the measurements $U_{s}(\mathbf{r} ; z=0)$ and $F$, and it can be directly or iteratively inverted to obtain $F$. It is equivalent to the $3 \mathrm{D} k$ space of the object filled with the 2D Fourier transform of the measured fields over the Ewald sphere. In terms of computational implementation, this mapping, Eq. (6), requires interpolation in the Fourier domain, and it can cause pixelation errors [28,29]. To avoid such errors, we directly define the single-scattering model in the spatial domain using Eq. (3). Specifically, we can propagate $U_{s}(\mathbf{r} ; z=0)$ to the plane located outside of the sample at $z=d$ using the propagation kernel [30]. The scattered field can be expressed in terms of the scattering potential using the Green's function resulting in

$U_{s}(\mathbf{r} ; z=d)=\int_{V} F\left(\mathbf{r}^{\prime}\right) U_{i}\left(\mathbf{r}^{\prime}\right) G\left(\mathbf{r} ; z=d-\mathbf{r}^{\prime}\right) d \mathbf{r}^{\prime}$.

Throughout this paper, we simulate the projections measured experimentally using the single-scattering propagation model of Eq. (7). We use this information to compare with the quantity, $U_{i}(\mathbf{r}) \log \left\{[U(\mathbf{r})] /\left[U_{i}(\mathbf{r})\right]\right\}$, which is necessity for the implementation of the Rytov reconstruction algorithm.

\section{Multiple-scattering propagation model (BPM)}

We evaluate the performance of ODT with a nonlinear propagation model based on the BPM [23]. We can propagate the light through an inhomogeneous medium by splitting the process in multiple fine steps, where each step consists of diffraction followed by refraction. Denoting the slowly varying envelope of the wave as $A(\mathbf{r})$, the total field can be written as $U(x, y, z)=A(x, y, z) e^{i k z}$. The BPM can be written as follows:

$$
\begin{aligned}
A(x, y, z+d z)= & e^{i k_{0} \Delta n(x, y, z)[d z /(\cos \theta)]} F_{2 \mathrm{D}}^{-1}\left\{F_{2 \mathrm{D}}[A(x, y, z)]\right. \\
& \left.\times e^{-i d z\left[\left(k_{x}^{2}+k_{y}^{2}\right) /\left(k+k_{z}\right)\right]}\right\}
\end{aligned}
$$

where $\Delta n$ is the contrast between the RI of the sample $[n(x, y, z)]$ and $n_{0}, k_{z}=\sqrt{k^{2}-k_{x}^{2}-k_{y}^{2}} . \theta$ is the angle of the illuminating beam as $\cos ^{-1}\left[\left(k_{z}^{\text {in }}\right) / k\right]$, given $\mathbf{k}^{\text {in }}=$ $\left(k_{x}^{\text {in }}, k_{y}^{\text {in }}, k_{z}^{\text {in }}\right)$ [31]. Again, the 2D Fourier-transform operator $\left(F_{2 \mathrm{D}}\right)$ and the 2D inverse Fourier-transform operator $\left(F_{2 \mathrm{D}}^{-1}\right)$ are defined as $F_{2 \mathrm{D}}[a(x, y, z)]=$ $\iint a(x, y, z) e^{-i\left(k_{x} x+k_{y} y\right)} d x d y$ and $F_{2 \mathrm{D}}^{-1}\left[\tilde{a}\left(k_{x}, k_{y}, z\right)\right]=\left[1 /(2 \pi)^{2}\right]$ $\iint \tilde{a}\left(k_{x}, k_{y}, z\right) e^{-i\left(k_{x} x+k_{y} y\right)} d k_{x} d k_{y}$, respectively [23,32].

\section{Iterative reconstruction algorithm}

Once the propagation model is determined as either a single-scattering or a multiple-scattering propagation model, we can specify a cost function that combines an error term and a regularization that incorporates prior knowledge regarding the sample. We impose the total-variation (TV) and non-negativity constraints [23]. Specifically, the cost function is defined as

$C(f)=\frac{1}{2 L} \sum_{l=1}^{L}\left\|g^{(l)}-A^{(l)}(f)\right\|_{2}^{2}+\tau D(f)+N(f)$,

where $g^{(l)} \in \mathbb{C}^{M}$ denotes the experimental measurements for a certain illumination angle $l, f \in \mathbb{R}^{N}$ denotes the object function (the RI contrast), $A^{(l)}: \mathbb{R}^{N} \rightarrow \mathbb{C}^{M}$ is the propagation model which can be either single scattering or multiple scattering for a certain angle $l, D(f)=$ $\sum_{n=1}^{N} \sqrt{\left(\nabla_{x} f\right)^{2}+\left(\nabla_{y} f\right)^{2}+\left(\nabla_{z} f\right)^{2}}\left(\nabla_{x}, \nabla_{y}\right.$, and $\nabla_{z}$ are

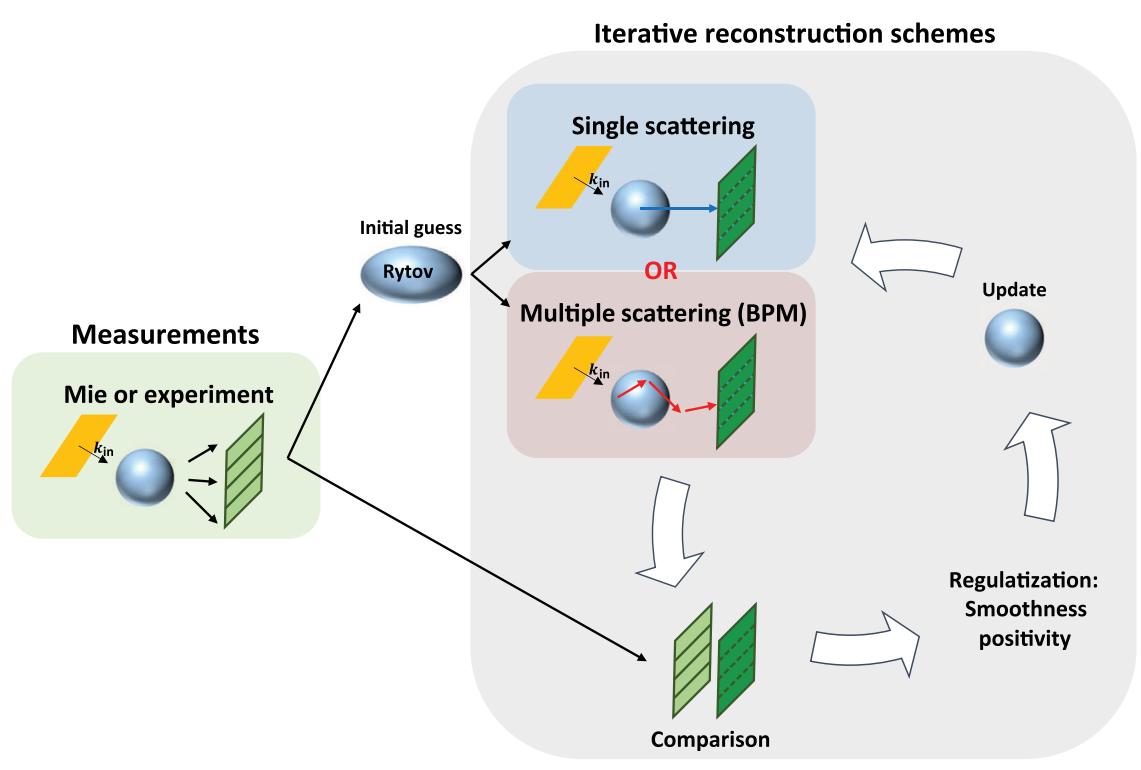

FIG. 1. The overall scheme of the paper. 
finite-difference operators in the $x, y$, and $z$ dimensions, respectively), $N(f)$ is the indicator function, $N(f)=$ $\infty(f<0)$ or $N(f)=0(f \geq 0)$, and $\tau$ is the regularization parameter setting the relative weight of the regularization term. To minimize the cost function, Eq. (9), in both cases, we use the fast iterative shrinkage-thresholding algorithm (FISTA) [24].

The overall scheme of the paper is summarized in Fig. 1. Measurements are acquired using either the Mie simulation or the experiment. The measurements are used in two ways. It is used to generate the initial guess (Rytov) via direct inversion and also to compare with estimated measurements generated by propagation models (single scattering or multiple scattering). As long as the error between the estimated and true measurements is large, we iteratively update the RI contrast while also exploiting prior knowledge (smoothness and positivity) in regularizations until the solution converges.

\section{METHOD}

\section{A. Simulation setup}

To obtain the equivalent experimental measurements, we use Mie theory to derive the scattered field by a single sphere $[25,26]$. In the simulations, the sample is illuminated at 95 different angles. The $k$ space representation of illumination angles is shown in Fig. 2. We test the algorithms on the following three different schemes. The first is the case of variable RI contrast with a fixed size (case 1), the second is the case of variable size with a fixed RI contrast (case 2), and the third is the case of differing RI contrast and size with a fixed sample-induced phase delay (case 3). We also investigate the ability of LT in handling multiple scattering using Mie theory for multiple cylinders [26]. Uniformly distributed 101 angles (three cylinders) or 161 angles (two cylinders) between $-\pi / 4$ and $\pi / 4$ are used

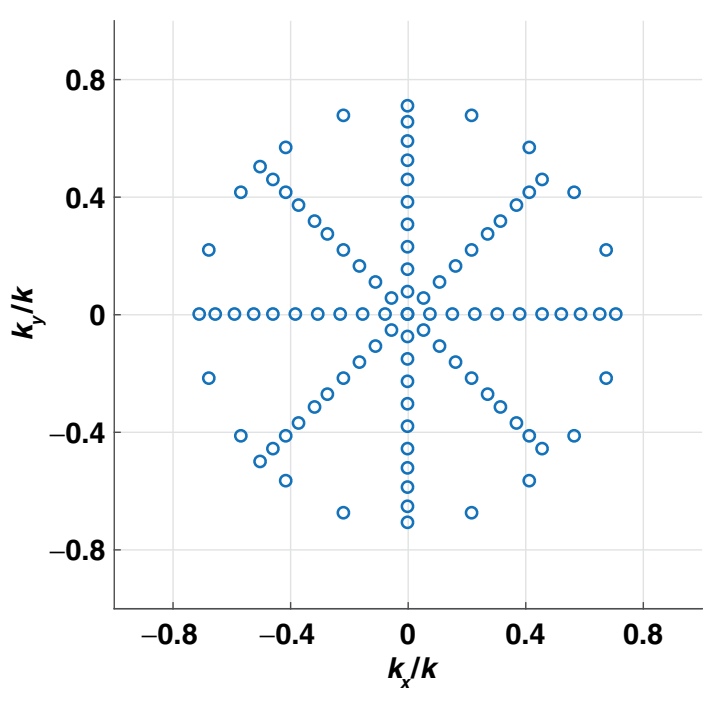

FIG. 2. $k$ space representation of normalized illumination $k$ vectors. to scan the samples. To quantitatively evaluate the performance, we calculate the error defined as

$$
\operatorname{error}\left(n_{\text {recon }}, n_{\text {true }}\right)=\frac{\left\|n_{\text {recon }}-n_{\text {true }}\right\|_{2}^{2}}{\left\|n_{\text {true }}-n_{0}\right\|_{2}^{2}},
$$

where $n_{\text {recon }}$ is the reconstructed solution (RI) and $n_{\text {true }}$ is the ground truth (RI) used in the Mie simulation. The error metric accounts for discrepancies in the shape of the object as well as in the index values. In addition, to compare the relative performances of the two propagation models, the relative error is used and is defined as

relative error $\left(n_{\mathrm{sgl}}, n_{\mathrm{mlp}}, n_{\text {true }}\right)=\frac{\operatorname{error}\left(n_{\mathrm{sgl}}, n_{\text {true }}\right)}{\operatorname{error}\left(n_{\mathrm{mlp}}, n_{\text {true }}\right)}$,

where $n_{\mathrm{sgl}}$ and $n_{\mathrm{mlp}}$ are solutions acquired from the singlescattering and multiple-scattering propagation models, respectively.

\section{B. Experimental setup}

The experimental apparatus consists of a Mach-Zehnder interferometer [20]. The signal and reference arms are recombined at an angle of $1.43^{\circ}$ before the detector in order to record off-axis digital holograms. The light source is a continuous-wave laser diode at $406 \mathrm{~nm}$ with a coherence length of $250 \mu \mathrm{m}$. The light is spatially filtered and collimated. A sample is placed in the signal arm between two infinity-corrected $100 \times$ oil-immersion microscope objectives (UPlanApo NA1.4 on the detection side and UplanFI NA1.3 on the illumination side). The effective numerical aperture of the system is 1.3. The image is projected on a scientific CMOS camera (Andor Neo) with an effective magnification of 111. The samples are illuminated with plane waves through the illumination objective at different incidence angles. Angular scanning of illumination is achieved by placing two galvo mirrors (one for the $x$ axis and the other one for the $y$ axis) in conjugate image planes of the sample.

In this paper, we consider three types of samples. The first sample consists of two silica fibers tapered down to a diameter of $9 \mu \mathrm{m}$ using a hot flame. The fibers are placed between the two objectives attached to an independent three-axis stage so that they could be moved with respect to each other. No coverslip is used in this case and the gap between the two objectives is filled with immersion oil. For the fiber experiment, the light source is a laser diode at a wavelength of $450 \mathrm{~nm}$. The second sample is a pair of polystyrene microspheres with a nominal diameter of $4.45 \mu \mathrm{m}$. The spheres are placed on two $150-\mu \mathrm{m}$-thick glass coverslips facing each other, with each sphere sitting on a different coverslip. The gap between the coverslips is filled with a low fluorescence immersion oil (Nikon type N, $n 0=1.518$ at $546.1 \mathrm{~nm}$ and $v e=41$ ). The coverslip holding the second sphere (close to the detector) can be moved and aligned with respect to the first bead using a 
piezoelectric-driven stage (PI instruments). The third sample is a pair of live yeast cells immersed in water. The cells are placed and controlled in the same way using the piezoelectric-driven stage so that they overlap in the $z$ axis. We acquire 160 views equally spaced either on a line along the $x$ axis when the two fibers are aligned along the $y$ axis or on a circle for the two beads and two cells in the $k_{x, y}$ plane at an angle of $42^{\circ}$ from the optical axis (the effective angle on the sample).

\section{Reconstruction setup}

The algorithms are implemented using custom scripts in MATLAB R2017a (MathWorks, Natick, Massachusetts) on a desktop computer (Intel Core i7-6700 CPU, 3.4 GHz, 32 gigabytes of RAM). For faster computation, a graphics processing unit (GeForce GTX 1070) is utilized. The computational space is sampled with step size $(\Delta x=\Delta y=\Delta z)$ of $0.08 \mu \mathrm{m}$ (single bead and three cylinders) or $0.0856 \mu \mathrm{m}$ (two fibers, two beads, and two cells). The regularization parameter, $\tau$, is manually set as described in Table I. For both models, the step size is reduced after every iteration, $\gamma^{k+1}=$ $0.985 \gamma^{k}$ (single sphere, two beads, and two cells) or $\gamma^{k+1}=$ $0.99 \gamma^{k}$ (multiple cylinders), and the iteration numbers for TV and FISTA are 20 and 200, respectively. For both propagation models, the stochastic gradient method is used with eight randomly chosen angles out of the total recorded angles at each iteration. In each case, the reconstruction obtained with direct inverse scattering based on the first-order Rytov approximation is used as the initial condition. The computational times of each model for various samples are also provided in Table I.

TABLE I. Parameters.

\begin{tabular}{lccc}
\hline \hline Data $($ single type) Size & & Single & Multiple \\
$(X \times Y \times Z)$ & Parameter & scattering & scattering \\
\hline Single sphere & $\gamma$ & 0.001 & 0.001 \\
$(350 \times 350 \times 128)$ & $\tau$ & 0.3 & 0.1 \\
Three cylinders & Time $(\mathrm{sec})$ & 1204 & 1218 \\
$(1024 \times 256)$ & $\gamma$ & 0.001 & 0.001 \\
& $\tau$ & 0.3 & 0.1 \\
Two cylinders $($ Mie $)$ & Time $(\mathrm{sec})$ & 90 & 217 \\
$(1024 \times 512)$ & $\gamma$ & 0.001 & 0.001 \\
& $\tau$ & 2 & 2 \\
Two cylinders $($ experiment $)$ & $\gamma$ & 179 & 411 \\
$(1024 \times 512)$ & $\tau$ & 2 & 0.005 \\
& Time $(\mathrm{sec})$ & 0.005 \\
Two beads & $\gamma(\mathrm{sec})$ & 174 & 409 \\
$(256 \times 256 \times 256)$ & $\gamma$ & 0.001 & 0.001 \\
& $\tau$ & 3 & 1.5 \\
Two cells & Time $(\mathrm{sec})$ & 989 & 1902 \\
$(256 \times 256 \times 256)$ & $\gamma$ & 0.001 & 0.001 \\
& $\tau$ & 0.01 & $10^{-5}$ \\
\hline \hline & Time $(\mathrm{sec})$ & 993 & 1946 \\
\hline
\end{tabular}

(a) Real $\left\{\log \left[U(\mathbf{r}) / U_{i}(\mathbf{r})\right]\right\}$
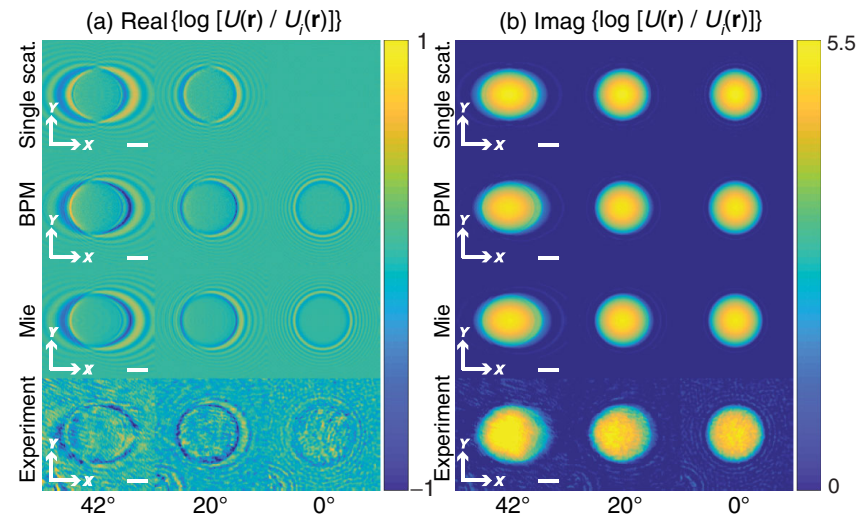

FIG. 3. (a),(b) Log of amplitude and phase images of a single bead. The first through fourth rows display the single-scattering, BPM, Mie, and experimental data. The first through third columns show illumination angles of $42^{\circ}, 20^{\circ}$, and $0^{\circ}$. The scale bars are $2 \mu \mathrm{m}$.

\section{RESULTS}

We simulate the two propagation models (the singlescattering model and the BPM) and then compare them with the two methods (Mie and experiment) with which we generate the projection data. Figure 3 shows the log of amplitude and the phase of the 2D projections obtained by different methods for different illumination angles. Log-ofamplitude and phase maps can be obtained by taking the real (log of amplitude) or imaginary (phase) part of $\log \left\{[U(\mathbf{r})] /\left[U_{i}(\mathbf{r})\right]\right\}$. For simulations (single scattering, BPM, and Mie), a $5-\mu \mathrm{m}$ bead $(n=1.605)$ is used with immersion oil $\left(n_{0}=1.537\right)$.

Looking at the experimental data in Fig. 3(a), we can observe that a dark rim around the sample becomes pronounced as the illumination angle increases. In addition, Mie shows more fluctuation in the amplitude maps with an increase in angle. The patterns of the dark rim and the fluctuation are more similar to the BPM than to the singlescattering model. For the phase maps, both single scattering and the BPM are similar to Mie and the experiment, as shown in Fig. 3(b). The sample-induced phase map becomes elongated along the illumination direction.

\section{A. Case 1: Variable RI contrast with a fixed size}

Case 1 shows the effect of RI contrast on the performance of each propagation model. Both models show a gradual distortion with the increase of RI contrast in Fig. 4 ( $Y Z$ cross sections of RI tomograms are provided in Appendix A, Fig. 17). To be specific, with the increase of RI contrast, the single-scattering model results in a lower estimation of RI values and distorted RI profiles. The multiple-scattering model shows a slight overestimation of RI values but more consistent profiles. The performance of each model is quantified by the error curve as in Fig. 5(a), showing lower errors of the multiple-scattering model for 


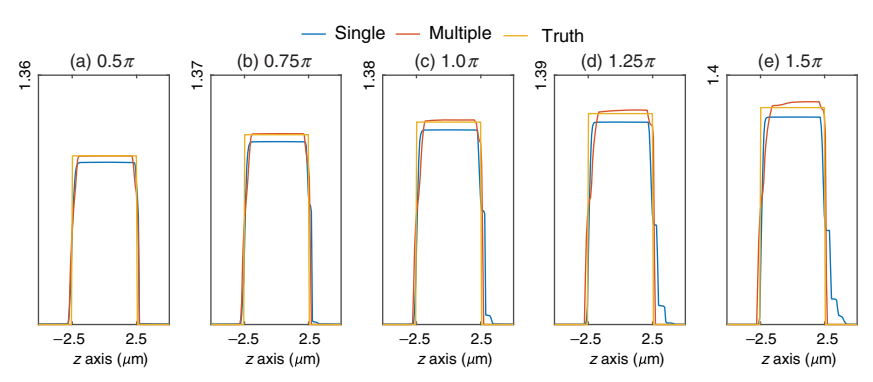

FIG. 4. $\quad z$ axis profiles of RI tomograms of a single bead using two different propagation models. From (a)-(e), the RI contrast increases proportionally so that the sample-induced phase delay equals to the number written on each column.
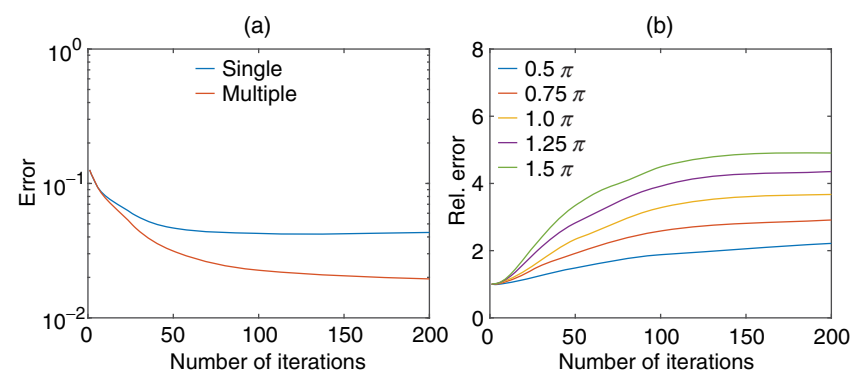

FIG. 5. (a) Errors of the single-scattering and multiple-scattering models for a $5-\mu \mathrm{m}$ bead inducing $0.5 \pi$ phase delay. (b) Relative errors for various beads which differ in the RI contrast.

all cases (for the other cases, errors are in Appendix B, Fig. 20). The relative effect of the increase in RI contrast can be investigated by looking at relative errors [Fig. 5(b)]. As the RI contrast gradually increases, relative errors increase in the range $0.5 \pi-1.5 \pi$. This result indicates that the multiple-scattering model breaks more slowly than the single-scattering model given the RI contrast changes.

\section{B. Case 2: Variable size with a fixed RI contrast}

For case 2, we assess the validity of the models by increasing the diameter of the sphere. It can be clearly observed that the reconstruction results from the singlescattering model are lower in the RI values than the ground

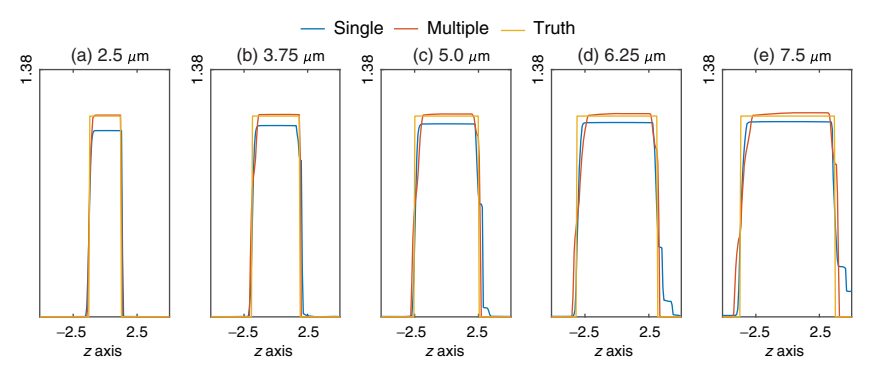

FIG. 6. $\quad z$ axis profiles of RI tomograms of a single bead using two different propagation models. From (a)-(e), the diameter increases proportionally. Each diameter is written on each column.
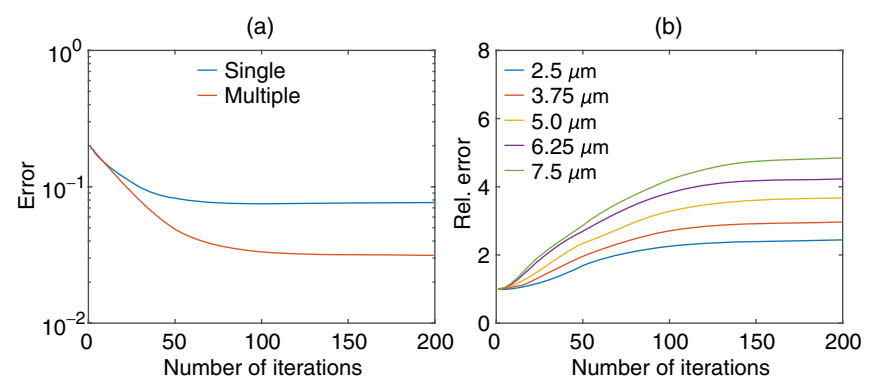

FIG. 7. (a) Errors of the single-scattering and multiple-scattering models for a $2.5-\mu \mathrm{m}$ bead inducing a $0.5 \pi$ phase delay. (b) Relative errors for various beads which differ in diameter.

truth but accurate in shapes. With an increase of the diameter, the RI values increase but distortions along the optical axis become pronounced, as shown in Fig. 6 ( $Y Z$ cross sections of RI tomograms are provided in Appendix A, Fig. 18). Compared to single scattering tomography, LT shows a good estimation of values overall and shows fewer distortions in shape. We observe lower error values than those of the single-scattering model [Fig. 7(a); for the other cases, the errors are in Appendix B, Fig. 21]. The advantage of LT against the single-scattering tomography can be clearly seen in Fig. 7(b). We can observe the gradual increase in relative-error values with an increase of the diameter, indicating the gradual breakdown of the singlescattering model is faster than the one of the multiplescattering model.

\section{Case 3: Different RI contrast and size with a fixed sample-induced phase delay}

The purpose of case 3 is to see the relation between the two different factors, the RI contrast and the size of the sample. Here, the size of the sphere varies, keeping the sample-induced phase delay at $\pi$. Therefore, each sample differs in both RI contrast and size, but the product of the two factors remains the same. In Fig. 8(a), the single- and multiple-scattering models show an underestimation and an overestimation of the RI values, respectively ( $Y Z$ cross

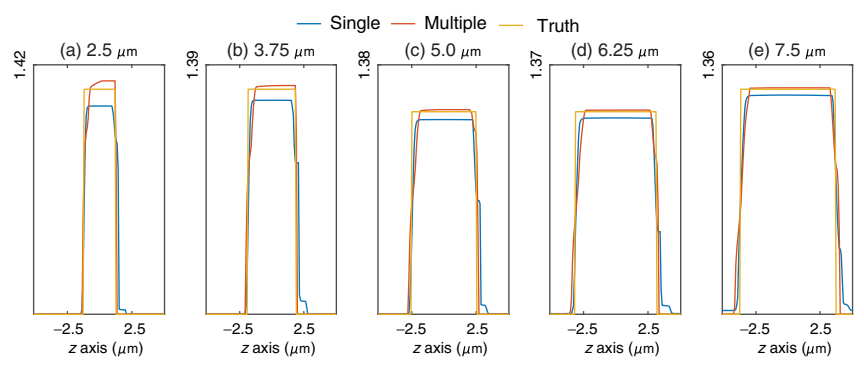

FIG. 8. $\quad z$ axis profiles of RI tomograms of a single bead using two different propagation models. From (a)-(e), the diameter increases proportionally and the RI contrast decreases, keeping sample-induced phase delay at $\pi$. The diameter is written atop each column. 

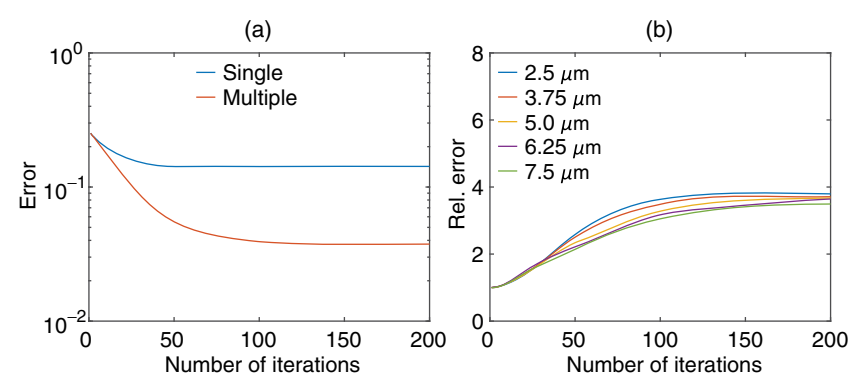

FIG. 9. (a) Errors of the single-scattering and multiple-scattering models for a $2.5-\mu \mathrm{m}$ bead inducing a $1 \pi$ phase delay. (b) Relative errors for various beads which differ in both the RI contrast and the diameter.

sections of RI tomograms are provided in Appendix A, Fig. 19). The inaccuracy decreases with an increase of the diameter (with a decrease of the RI contrast). However, at the same time, both models show more distortion in profiles with an increase of the diameter. In case 3, the increase in the diameter (the decrease in the RI contrast) has countereffects since the RI values become more accurate but the shapes become more inaccurate. To numerically analyze the results, the errors are calculated as in Fig. 9(a) (for the other cases, the errors are in Appendix B, Fig. 22). In case 3 , where the two effects have compensatory effects on the errors, it is possible to observe that the relative-error values converge [Fig. 9(b)] with the number of iterations. However, the fact that the points converge around the value of 4 indicates that LT is still more accurate than single-scattering tomography is.

\section{Comparative analysis}

The converging points, which are defined as the end points of errors and relative errors after 200 iterations, are plotted in Fig. 10. Figures 10(a), 10(c), and 10(e) give us more information about how the error of each model is affected by changes in the individual factor explaining changes in the relative errors in Figs. 10(b), $10(\mathrm{~d})$, and 10(f). Overall, the breakdown of the singlescattering propagation model is faster than the one of the multiple-scattering propagation model with either an increase of the RI contrast or the size, as you can see in Figs. 10(b) and 10(d). When both the RI contrast and the size change [Figs. 10(e) and 10(f)], the situation becomes more complex. The converging points of the relative errors stay almost constant, as shown in Fig. 10(f), because of the different effectiveness of each factor. To be specific, in terms of relative errors, when the RI contrast increases and the size decreases, the effect of each factor compensates for the opposite effect.

\section{E. Simulation of the reconstruction of three cylinders}

To test the capacity for handling multiple scattering, simulated measurements for multiple cylinders are
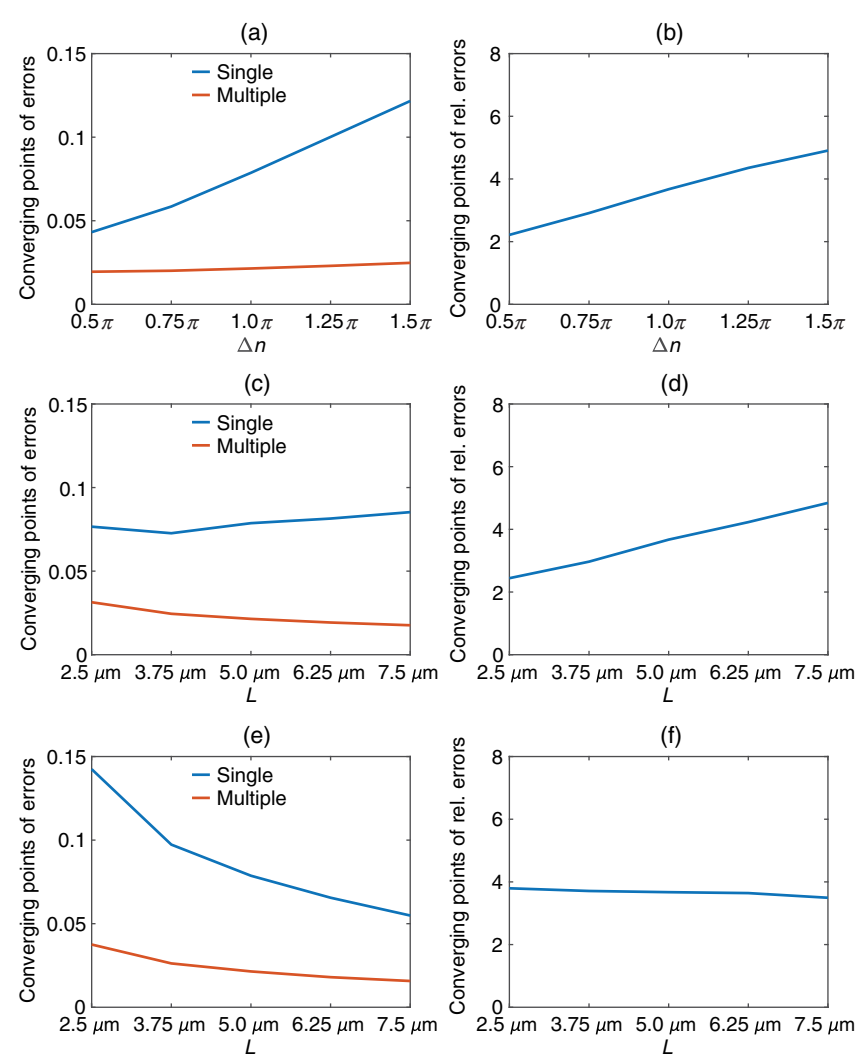

FIG. 10. Converging points of errors and relative errors for (a), (b) case $1,(\mathrm{c}),(\mathrm{d})$ case 2 , and (e),(f) case 3.

generated using Mie theory [26]. A set of three cylinders whose diameters are $4 \mu \mathrm{m}$ and whose RI contrasts are 0.0254 is considered. The set of cylinders is rotated from $0^{\circ}$ to $90^{\circ}$, resulting in the gradual increase of the multiplescattering effect. As shown in Fig. 11, we can clearly see that LT outperforms the single-scattering propagation model for all cases. While the single-scattering tomography shows smearing between cylinders, the LT maintains a clear distinction among the three cylinders. It can be quantitatively confirmed through the errors and relative errors (For the other cases, the errors are in Appendix A, Fig. 23), as shown in Fig. 12.

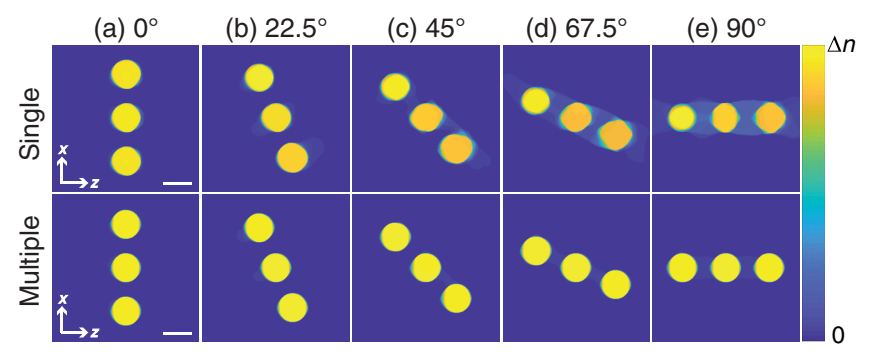

FIG. 11. $X Z$ cross sections of RI tomograms of three cylinders using two different propagation models. (Upper row) Single scattering. (Lower row) Multiple scattering. From (a)-(e), the sample is gradually rotated from $0^{\circ}$ to $90^{\circ}$. The scale bars are $4 \mu \mathrm{m}$ and $\Delta n$ is 0.0254 . 
(a)
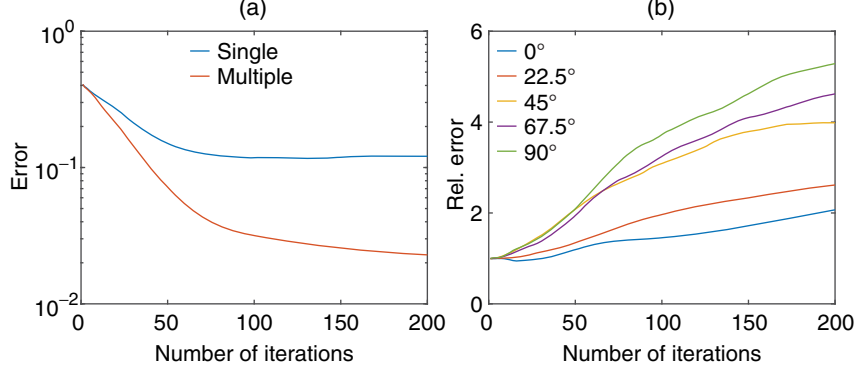

FIG. 12. (a) Error of the single-scattering and multiple-scattering models for multiple cylinders rotated $90^{\circ}$. (b) Relative errors for three cylinders rotated from $0^{\circ}$ to $90^{\circ}$.

\section{F. Phase-unwrapping simulations}

The Rytov algorithm that we use in this paper as the initial condition for the LT algorithm, and also as the core of the single-scattering model algorithm, relies on the measurement of the phase of each projection. If the optical thickness of the sample exceeds $2 \pi$, then the measured phase must be unwrapped. Here, we compare the performance of two propagation models when the phaseunwrapping algorithm fails. We perform simulations using Mie theory to simulate the 2D projections that are holographically recorded in the real system. The RI contrast is increased so that the sample-induced phase delay results in $3 \pi$. It is then difficult to properly unwrap the phase, even with a state-of-the-art algorithm such as the Phase Unwrapping Max-flow Algorithm, which is used throughout this paper [33]. Since the Rytov approximation requires unwrapped phases for the reconstruction, failure in unwrapping directly relates to severe distortions in Rytov reconstructions, as shown in Fig. 13(a). Accurate phase unwrapping is critical not only to single-scattering tomography but also to LT because phase-wrapped measurements result in undesirable local minima in the minimization algorithm. Compared to single-scattering tomography, which shows only smoothing effects [Fig. 13(b)], LT is able to successfully reconstruct the tomograms [Fig. 13(c)], even though it uses Fig. 13(a) as the initialization. The effect is more dramatic in terms of error, as in Fig. 13(d).

\section{G. Comparison of simulations to experiments \\ 1. Two fibers}

We place two polystyrene fibers in three different configurations, which are $0^{\circ}, 40^{\circ}$, and $90^{\circ}$, where $0^{\circ}$ indicates that the two fibers are placed side by side and $90^{\circ}$ indicates that they are one after the other along the light propagation axis $(z)$. Just as we compare the propagation models for a single sphere in Fig. 3, we compare the ones for two fibers placed in the different configurations and added in Appendix C. In Fig. 11, we conclude that LT is more robust to the rotation angle, showing clear distinctions among cylinders, than the single-scattering propagation model. This finding is experimentally confirmed again. In Fig. 14, the single-scattering model suffers from a gradual smearing between two fibers with an increase of the rotation angle. By contrast, LT shows not only two distinct fibers but also more consistency in the shapes and values of the fibers, regardless of the rotation angle. Furthermore, to confirm the experimental results, we simulate the same configurations by placing two fibers whose diameters are $9 \mu \mathrm{m}$ and $\Delta n \mathrm{~s}$ are -0.055 at three different rotation angles, $0^{\circ}, 40^{\circ}$, and $90^{\circ}$. It is possible to clearly observe not only the capacity of LT but also similar distortion patterns in the single-scattering model in Fig. 14. Therefore, the capacity of LT in handling multiple scattering is again confirmed.

\section{Two beads}

We prepare a sample consisting of two spherical beads so that we can experimentally record the holographic projections and use the data to compare two propagation models. Two $4.45-\mu \mathrm{m}$ beads are placed in a row so that they overlap in the $z$ axis. In the case of single-scattering tomography which is based on Rytov, the location of the image plane is very important [29]. Figure 11(c) confirms this fact. As the sample is placed farther from the image plane, RI tomograms become either smaller in size and higher in RI contrast or bigger in size and lower in RI contrast, depending on the direction of defocus. This result is confirmed again with two beads. It comes from the fact that the singlescattering propagation model based on Rytov causes distortions moving away from the focal plane, as reported in (a) Rytov

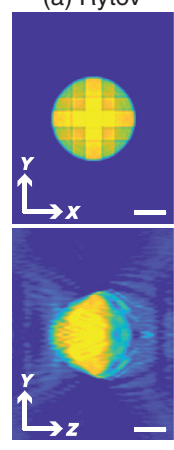

(b) Single

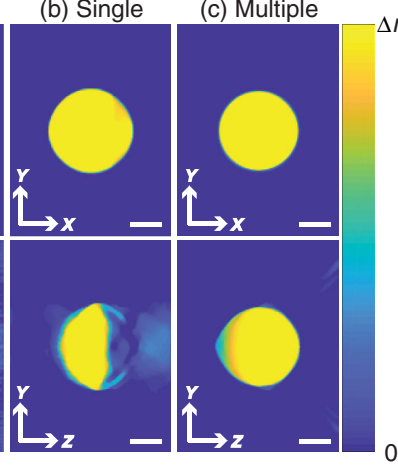

(d) $3 \pi$

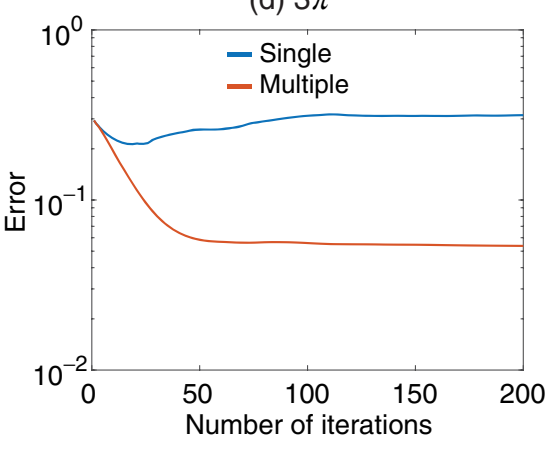

FIG. 13. $X Y$ and $Y Z$ cross sections of RI tomograms of a high-contrast bead from (a) Rytov approximation, (b) single-scattering tomography, and (c) LT. (d) Error of singlescattering and multiple-scattering propagation models. The scale bars are $2 \mu \mathrm{m}$ and $\Delta n$ is 0.1218 . 
(a) $0^{\circ}$

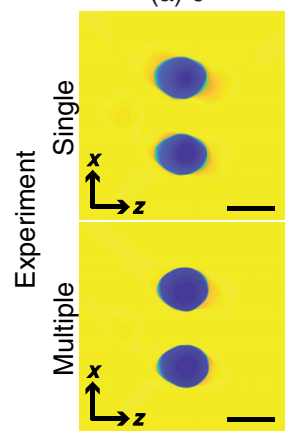

(b) $40^{\circ}$

(c) $90^{\circ}$

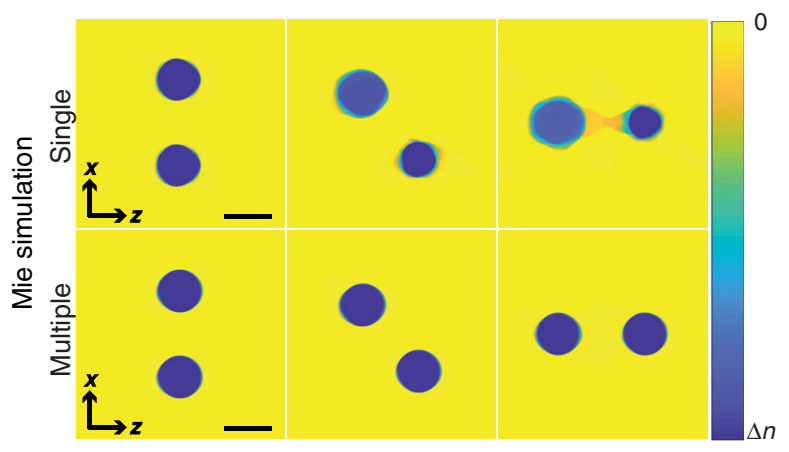

FIG. 14. $\quad X Z$ cross sections of RI tomograms of two fibers using two different propagation models. (Upper row) Single scattering. (Lower row) Multiple scattering. From (a)-(c), the sample is rotated for three angles, $0^{\circ}, 40^{\circ}$, and $90^{\circ}$. The scale bars are $10 \mu \mathrm{m}$ and $\Delta n$ is -0.055 .

Ref. [29]. As shown in Figs. 15(a)-15(c), the image obtained before the image plane becomes smaller in size and higher in RI contrast, and the one after the image plane becomes bigger in size and lower in RI contrast. By contrast, LT clearly reconstructs two beads of equal size and contrast [Figs. 15(d)-15(f)]. Since the BPM

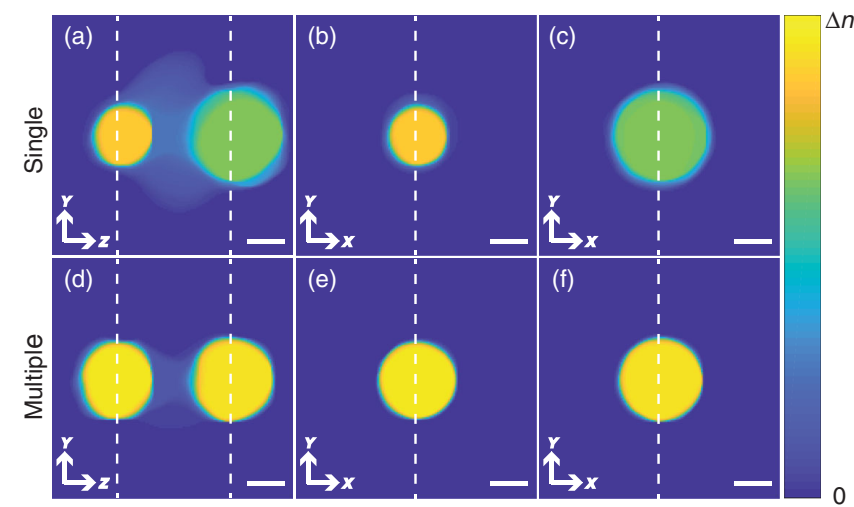

FIG. 15. (a),(b) $Y Z$ and (b),(e) $X Y$ (left bead), and (c),(f) $X Y$ (right bead) cross sections of RI tomograms of two beads using different propagation models. (Upper row) Single scattering. (Lower row) Multiple scattering. The white dotted lines represent slices of the complementary figures. The scale bars are $2 \mu \mathrm{m}$ and $\Delta n$ is 0.0894 .

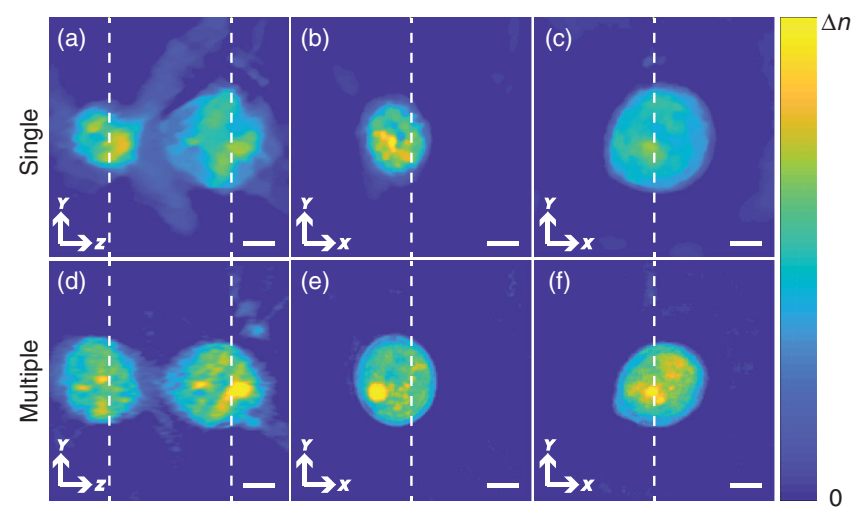

FIG. 16. (a),(b) $Y Z$, (b),(e) $X Y$ (left cell), and (c),(f) $X Y$ (right cell) cross sections of RI tomograms of two cells using different propagation models. (Upper row) Single scattering. (Lower row) Multiple scattering. The white dotted lines represent the slices of the complementary figures. The scale bars are $2 \mu \mathrm{m}$ and $\Delta n$ is 0.125 .

describes the propagated field itself through the sample, it is not only unaffected by the location of the image plane but also able to handle multiple scattering.

\section{Yeast cells}

To confirm the capability of LT with complex objects, we also apply LT on biological cells rather than on the samples whose RI values are homogeneous. Two yeast cells are placed in a row, so the overall configuration is similar to the previous two-bead case, but the cells have more complex internal structures. Since the samples have more fine structures, it restricts us from using a high regularization parameter, making the cost function more dependent on the propagation models. When looking at the cells in Fig. 16, the one before the focal plane becomes smaller [Fig. 16(b)] and the other grows larger [Fig. 16(c)]. This distortion has been already observed in the two-bead case, Fig. 15. This problem becomes more severe when a sample has fine structures because many structures out of the focal plane are differently distorted depending on the distance from the focal plane and because the distorted parts interact with one another. Therefore, the resolution deteriorates. By contrast, LT tomography which makes use of the electric field itself does not suffer from the problem, showing clear cell structures even out of the focal plane, as shown in Figs. 16(d)-16(f).

\section{CONCLUSION}

In this paper, we rigorously compare LT against conventional single-scattering tomography. Mie theory provides the analytical solution for the scattered field, given a sphere so that we are able to evaluate the reconstruction fidelity of each model accurately. To investigate the capability of each model in dealing with nonlinearity, two factors which 
are directly related to nonlinearity, the RI contrast and the size, are controlled either independently or simultaneously. In all of the cases, LT consistently outperforms singlescattering tomography. We attribute this improvement to the fact that the BPM used by LT captures multiple forward-scattering events. In general the BPM performs relatively well in the simulation of inhomogeneous media with small index contrast since the main limitation of the method is the assumption that reflections can be neglected. The improvement in performance becomes more pronounced as the index or the diameter of the beads increases. The most dramatic improvement is observed when we image multiple objects (three cylinders). This result confirms the observation that object size matters since we can consider the set of three cylinders a single large object. For samples whose optical path exceed $2 \pi$, phase unwrapping must be deployed in the Rytov algorithm. When the phaseunwrapping algorithm fails as the optical path across the $5-\mu \mathrm{m}$ sphere increases to $3 \pi$, single-scattering tomography becomes severely distorted. For this case, we observe that the iterations of the LT algorithm are able to correct the distortions that are evident in the Rytov reconstruction due to phase-unwrapping limitations. Finally, the reconstruction of the Rytov algorithm is in focus only at the plane of best focus of the optical system. For thick samples, the sample becomes blurry away from the focal plane. This defocusing is evident in Fig. 14. The distortion is a combination of two factors: defocusing and multiple scattering. The BPM helps to alleviate both of these problems, allowing us to keep the entire sample in focus. This result is experimentally confirmed using various samples, two fibers, two beads, and two cells. As the sample becomes more complex (thicker and with a higher index contrast), ultimately, the BPM provides an inadequate estimate for the scattered field by the object since reflections are neglected and the vectorial nature of the optical field is ignored. In this case, the only way to realize an improvement in performance is by adopting a more sophisticated scattering model. There is an intermediate level of sample complexity, however, at which the BPM still provides a reasonably accurate prediction of the scattered field but at which the nonlinear inversion problem becomes very difficult due to the emergence of strong local minima. In this regime, we believe that there is a global minimum which is a good approximation of the true object, but the LT algorithm cannot find it. It is possible that more powerful optimization algorithms than the stochastic method we use in this paper can provide a significant improvement in performance.

\section{APPENDIX A: FIGURES OF RI TOMOGRAMS}

As supplementary figures, RI tomograms of a single bead are provided for each case.

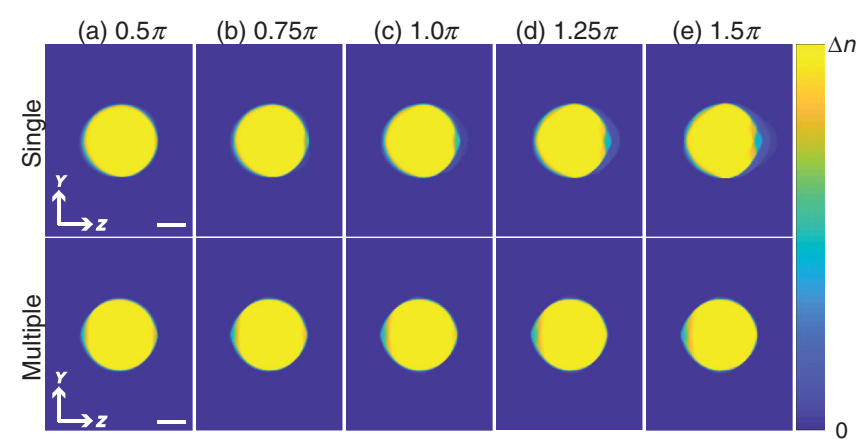

FIG. 17. $Y Z$ cross sections of RI tomograms of a single bead using two different propagation models. (Upper row) Single scattering. (Lower row) Multiple scattering. From (a)-(e), the RI contrast increases proportionally so that the sample-induced phase delay equals the number written atop each column. The scale bars are $2 \mu \mathrm{m}$ and $\Delta n=[($ phase delay $) / \pi] \times 0.0406$.

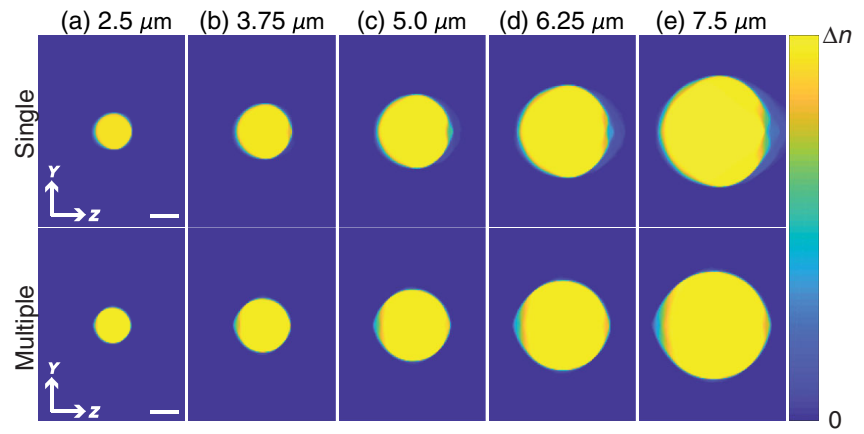

FIG. 18. $\quad Y Z$ cross sections of reconstructed RI tomograms of a single bead using two different propagation models. (Upper row) Single scattering. (Lower row) Multiple scattering. From (a)-(e), the diameter increases proportionally. The diameter is written atop each column. The scale bars are $2 \mu \mathrm{m}$ and $\Delta n$ is 0.0406 .

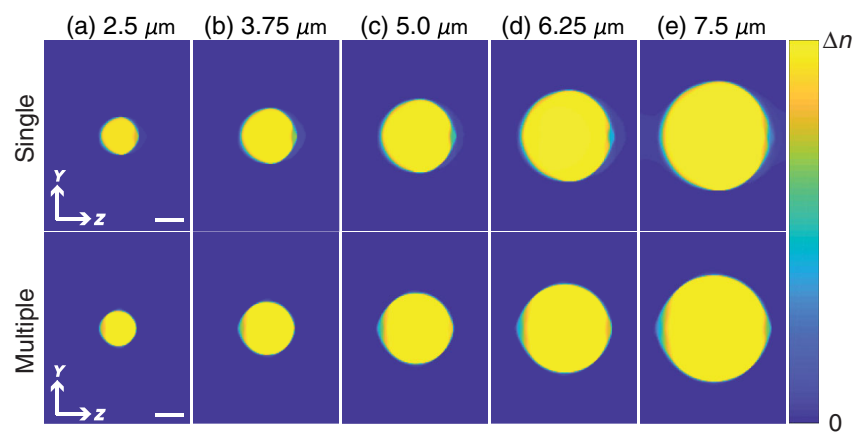

FIG. 19. $Y Z$ cross sections of RI tomograms of a single bead using two different propagation models. (Upper row) Single scattering. (Lower row) Multiple scattering. From (a)-(e), the diameter increases proportionally and the RI contrast decreases, keeping sample-induced phase delay at $\pi$. The diameter is written atop each column. The scale bars are $2 \mu \mathrm{m}$ and $\Delta n=[(5 \mu \mathrm{m}) /$ diameter $] \times 0.0406$. 


\section{APPENDIX B: FIGURES OF MODEL ERRORS}

As supplementary figures, the errors for the sample are provided in detail for each case.
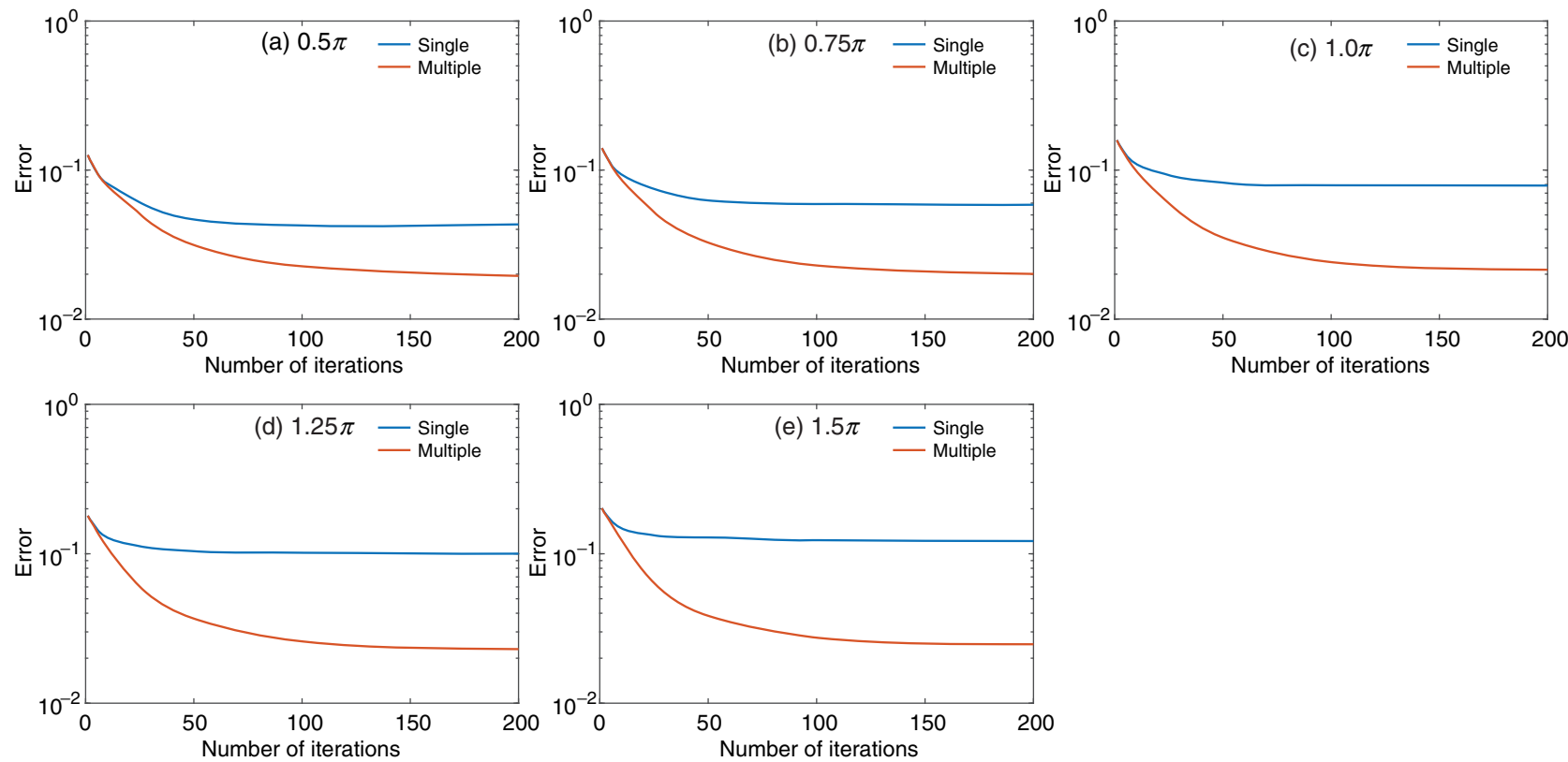

FIG. 20. Errors of the single-scattering and multiple-scattering models for various beads with differing RI contrasts. From (a)-(e), the RI contrast increases proportionally, so sample-induced phase delay equals the number written in each subfigure.
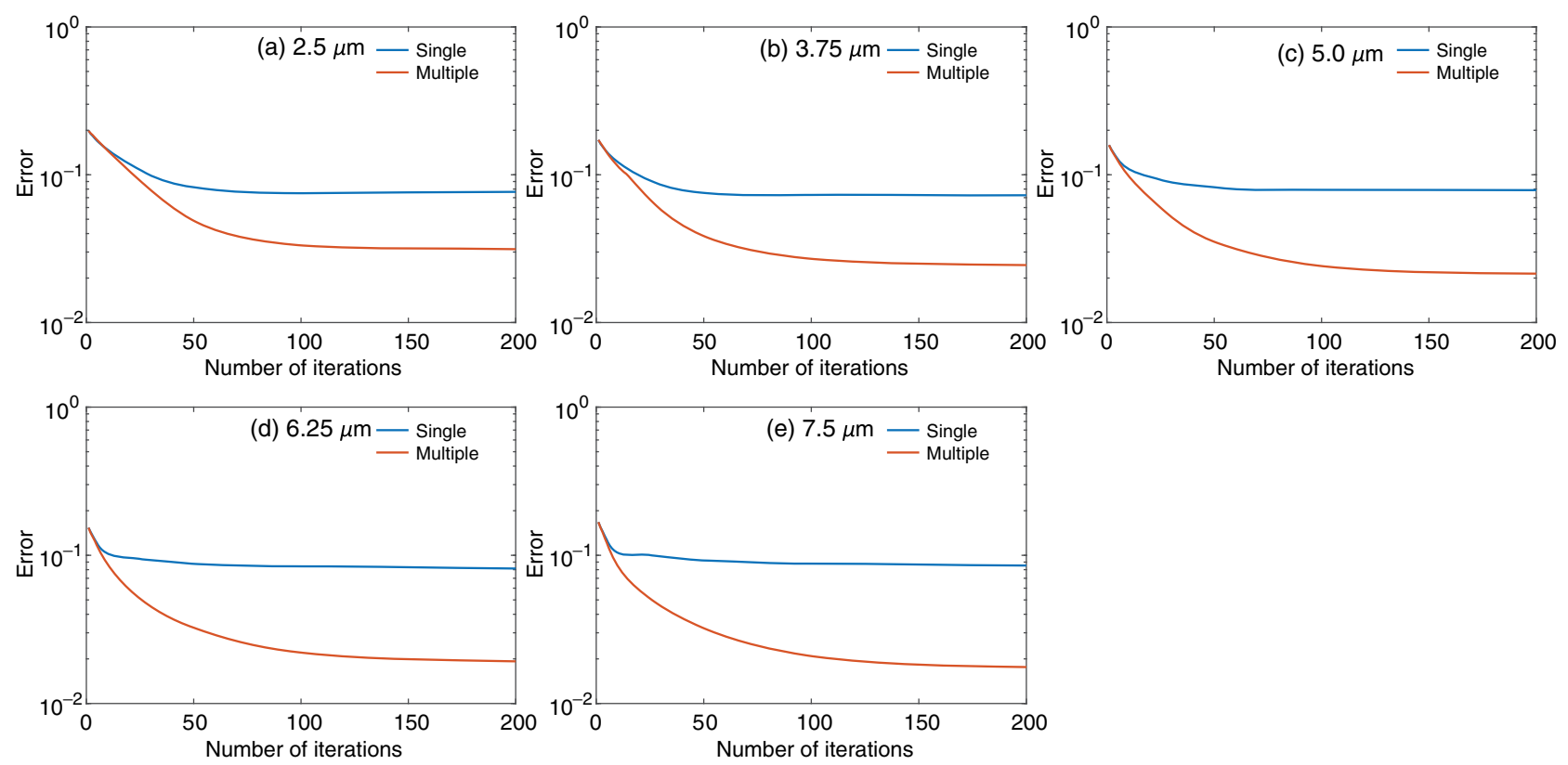

FIG. 21. Errors of the single-scattering and multiple-scattering models for various beads with differing diameters. From (a)-(e), the diameter increases proportionally. The diameter is written in each subfigure. 

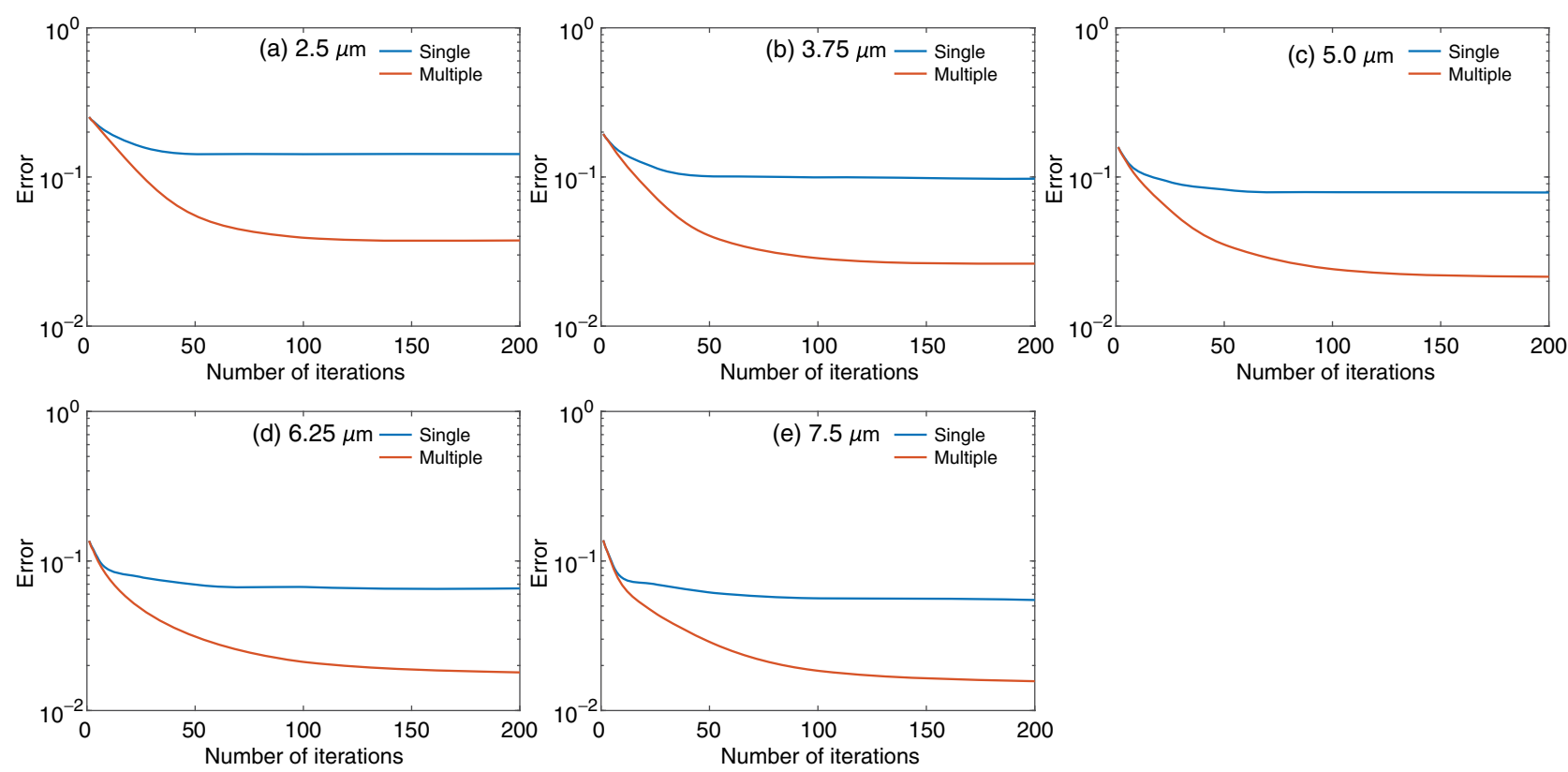

FIG. 22. Errors of the single-scattering and multiple-scattering models for various beads differing in both RI contrast and diameter. From (a)-(e), the diameter increases proportionally and the RI contrast decreases, keeping sample-induced phase delay at $\pi$. The diameter is written in each subfigure.
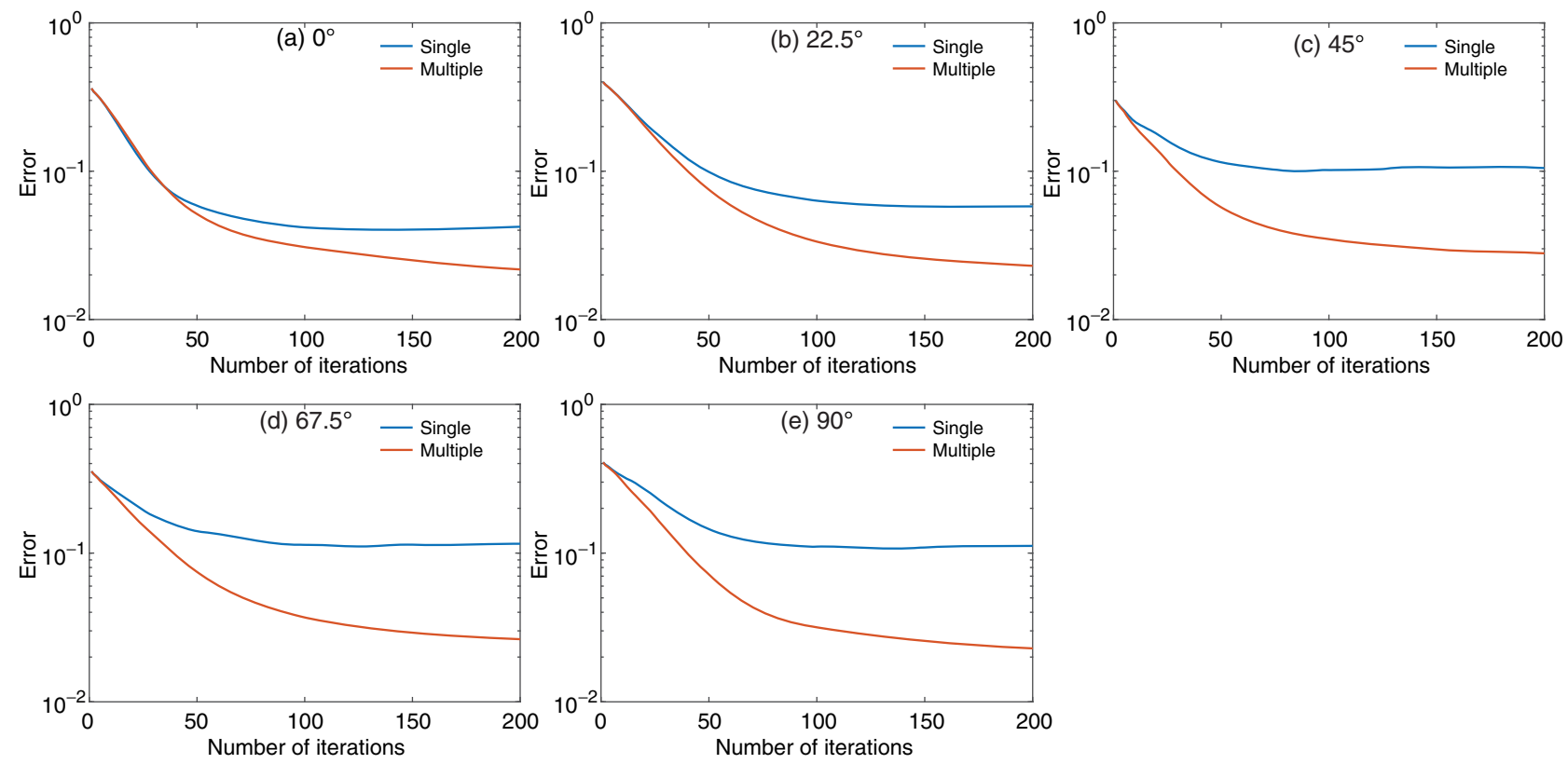

FIG. 23. Errors of the single-scattering and multiple-scattering models for three cylinders which are gradually rotated (a)-(e) $0^{\circ}-90^{\circ}$ with respect to the optical axis.

\section{APPENDIX C: COMPARISON OF PROPAGATION MODELS OF TWO FIBERS}

We simulate two different propagation models, singlescattering propagation and the BPM, and then compare them with Mie and experimental data for two fibers placed in different configurations. In Fig. 24, we can clearly see differences among the single-scattering model, Mie, and experimental data, especially in regions where the cylinders intersect. On the other hand, interestingly, the BPM shows a very similar log of amplitude and phase map to those of the Mie and experimental data. We attribute this finding to the capacity of the BPM to capture multiple scattering, which is confirmed through the following results. 
(a) Real $\left\{\log \left[\mathrm{U}(\mathbf{r}) / U_{i}(\mathbf{r})\right]\right\}$

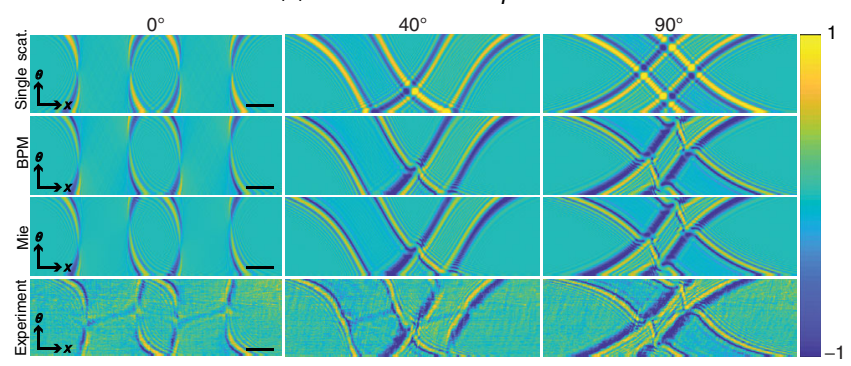

(b) Imag $\left\{\log \left[U(\mathbf{r}) / U_{i}(\mathbf{r})\right]\right\}$

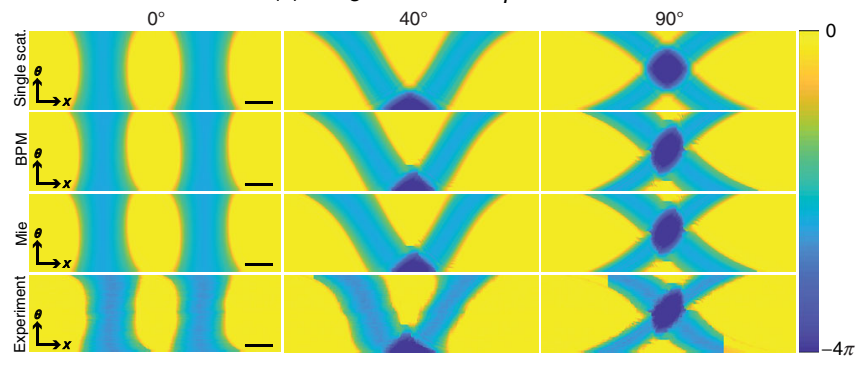

FIG. 24. Sinograms of log of (a) amplitude and (b) phase images of two fibers. The first through fourth rows indicate single-scattering, BPM, Mie, and experimental data. The first through third columns display rotation configurations of $0^{\circ}, 40^{\circ}$, and $90^{\circ}$. The scale bars are $5 \mu \mathrm{m}$.

\section{APPENDIX D: NOISE SIMULATION FOR THREE CYLINDERS}

We add shot noise to the interferograms. The SNR in decibels of shot noise can be defined as follows: $10 \log \sqrt{N}$, where $N$ is the number of photons per pixel. We generate shot noise on interferograms of the three cylinders in the paper from a Poisson distribution with a mean parameter of $N[I /\langle I\rangle]$, where $I$ is the intensity of the simulated interferogram and $\langle\cdots\rangle$ is the average. Figure 25 shows the converging points of errors for both noiseless and noisy cases. In noisy cases, we simulate two different cases of $N$, 5000 and 500. The converging points of the noisy cases are
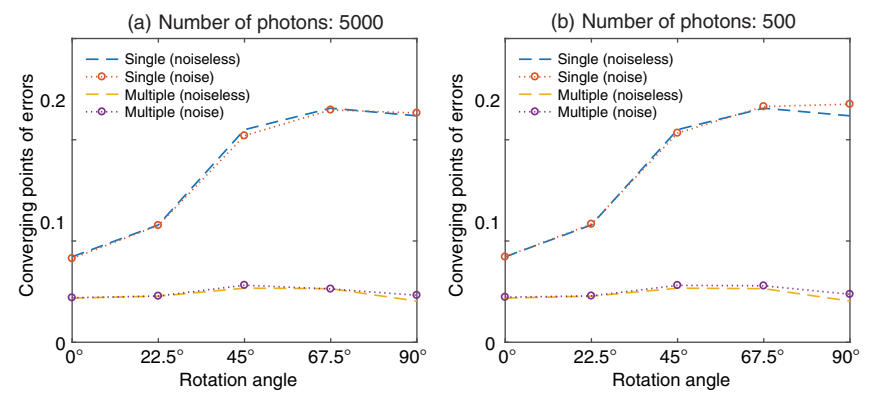

FIG. 25. Converging points of errors for three cylinders which are gradually rotated from $0^{\circ}-90^{\circ}$. Two different average numbers of photons per pixel, (a) 5000 and (b) 500, are used to generate the shot noise. not significantly different from the noiseless case. We can conclude that the iterative reconstruction scheme with TV regularization can handle the shot noise effectively unless the light level is very low.

[1] KyeoReh Lee, Kyoohyun Kim, Jaehwang Jung, JiHan Heo, Sangyeon Cho, Sangyun Lee, Gyuyoung Chang, YoungJu Jo, Hyunjoo Park, and YongKeun Park, Quantitative phase imaging techniques for the study of cell pathophysiology: From principles to applications, Sensors 13, 4170 (2013).

[2] Gabriel Popescu, Takahiro Ikeda, Ramachandra R. Dasari, and Michael S. Feld, Diffraction phase microscopy for quantifying cell structure and dynamics, Opt. Lett. 31, 775 (2006).

[3] YongKeun Park, Catherine A. Best, and Gabriel Popescu, in Mechanobiology of Cell-Cell and Cell-Matrix Interactions, edited by A. Wagoner Johnson and Brendan Harley (Springer, New York, 2011), p. 279.

[4] YongKeun Park, Toyohiko Yamauchi, Wonshik Choi, Ramachandra Dasari, and Michael S. Feld, Spectroscopic phase microscopy for quantifying hemoglobin concentrations in intact red blood cells, Opt. Lett. 34, 3668 (2009).

[5] JaeHwang Jung, Kyoohyun Kim, HyeonSeung Yu, KyeoReh Lee, SeoEun Lee, SeungHoon Nahm, HyunJoo Park, and YongKeun Park, Biomedical applications of holographic microspectroscopy [invited], Appl. Opt. 53, G111 (2014).

[6] R Barer, Interference microscopy and mass determination, Nature (London) 169, 366 (1952).

[7] Gabriel Popescu, YoungKeun Park, Niyom Lue, Catherine Best-Popescu, Lauren Deflores, Ramachandra R Dasari, Michael S. Feld, and Kamran Badizadegan, Optical imaging of cell mass and growth dynamics, Am. J. Physiol. Cell Physiol. 295, C538 (2008).

[8] Wonshik Choi, Christopher Fang-Yen, Kamran Badizadegan, Seungeun Oh, Niyom Lue, Ramachandra R. Dasari, and Michael S. Feld, Tomographic phase microscopy, Nat. Methods 4, 717 (2007).

[9] Taewoo Kim, Renjie Zhou, Mustafa Mir, S. Derin Babacan, P. Scott Carney, Lynford L. Goddard, and Gabriel Popescu, White-light diffraction tomography of unlabelled live cells, Nat. Photonics 8, 256 (2014).

[10] Di Jin, Renjie Zhou, Zahid Yaqoob, and Peter T. C. So, Tomographic phase microscopy: Principles and applications in bioimaging, J. Opt. Soc. Am. B 34, B64 (2017).

[11] Yongjin Sung and Ramachandra R. Dasari, Deterministic regularization of three-dimensional optical diffraction tomography, J. Opt. Soc. Am. A 28, 1554 (2011).

[12] Yongjin Sung, Wonshik Choi, Christopher Fang-Yen, Kamran Badizadegan, Ramachandra R. Dasari, and Michael S. Feld, Optical diffraction tomography for high resolution live cell imaging, Opt. Express 17, 266 (2009).

[13] JooWon Lim, KyeoReh Lee, Kyong Hwan Jin, Seungwoo Shin, SeoEun Lee, YongKeun Park, and Jong Chul Ye, Comparative study of iterative reconstruction algorithms for missing cone problems in optical diffraction tomography, Opt. Express 23, 16933 (2015). 
[14] Max Born and Emil Wolf, Principles of Optics: Electromagnetic Theory of Propagation, Interference and Diffraction of Light (Cambridge University Press, Cambridge, England, 1999), Chap. 13, pp. 695-734.

[15] Zachary A. Steelman, Will J. Eldridge, Jacob B. Weintraub, and Adam Wax, Is the nuclear refractive index lower than cytoplasm? Validation of phase measurements and implications for light scattering technologies, J. Biophoton. 10, 1714 (2017).

[16] Malcolm Slaney, Avinash C. Kak, and Lawrence E. Larsen, Limitations of imaging with first-order diffraction tomography, IEEE Trans. Microwave Theory Tech. 32, 860 (1984).

[17] Bingquan Chen and Jakob J. Stamnes, Validity of diffraction tomography based on the first Born and the first Rytov approximations, Appl. Opt. 37, 2996 (1998).

[18] Mani Azimi and A.C. Kak, Distortion in diffraction tomography caused by multiple scattering, IEEE Trans. Med. Imaging 2, 176 (1983).

[19] Lei Tian and Laura Waller, 3D intensity and phase imaging from light field measurements in an LED array microscope, Optica 2, 104 (2015).

[20] Ulugbek S. Kamilov, Ioannis N. Papadopoulos, Morteza H. Shoreh, Alexandre Goy, Cedric Vonesch, Michael Unser, and Demetri Psaltis, Learning approach to optical tomography, Optica 2, 517 (2015).

[21] Hsiou-Yuan Liu, Dehong Liu, Hassan Mansour, Petros T. Boufounos, Laura Waller, and Ulugbek S. Kamilov, SEAGLE: Sparsity-driven image reconstruction under multiple scattering, IEEE Trans. Comput. Imaging 4, 73 (2017).

[22] Katsunari Okamoto, Fundamentals of Optical Waveguides (Academic Press, New York, 2010).

[23] Ulugbek S. Kamilov, Ioannis N. Papadopoulos, Morteza H. Shoreh, Alexandre Goy, Cedric Vonesch, Michael Unser, and Demetri Psaltis, Optical tomographic image reconstruction based on beam propagation and sparse regularization, IEEE Trans. Comput. Imaging 2, 59 (2016).
[24] Amir Beck and Marc Teboulle, A fast iterative shrinkagethresholding algorithm for linear inverse problems, SIAM J. Imaging. Sci. 2, 183 (2009).

[25] Craig F. Bohren and Donald R. Huffman, Absorption and Scattering of Light by Small Particles (John Wiley \& Sons, New York, 2008).

[26] J. Schäfer, S.-C. Lee, and A. Kienle, Calculation of the near fields for the scattering of electromagnetic waves by multiple infinite cylinders at perpendicular incidence, J. Quant. Spectrosc. Radiat. Transfer 113, 2113 (2012).

[27] Justin D. Keener, Kevin J. Chalut, John W. Pyhtila, and Adam Wax, Application of mie theory to determine the structure of spheroidal scatterers in biological materials, Opt. Lett. 32, 1326 (2007).

[28] S. Pan and A. Kak, A computational study of reconstruction algorithms for diffraction tomography: Interpolation versus filtered-backpropagation, IEEE Trans. Acoust. Speech Signal Process. 31, 1262 (1983).

[29] Julianna Kostencka, Tomasz Kozacki, Arkadiusz Kuś, Björn Kemper, and Małgorzata Kujawińska, Holographic tomography with scanning of illumination: Space-domain reconstruction for spatially invariant accuracy, Biomed. Opt. Express 7, 4086 (2016).

[30] Wonshik Choi, Christopher Fang-Yen, Kamran Badizadegan, Ramachandra R. Dasari, and Michael S. Feld, Extended depth of focus in tomographic phase microscopy using a propagation algorithm, Opt. Lett. 33, 171 (2008).

[31] Yijun Bao and Thomas K. Gaylord, Clarification and unification of the obliquity factor in diffraction and scattering theories: Discussion, J. Opt. Soc. Am. A 34, 1738 (2017).

[32] M. D. Feit and J. A. Fleck, Beam nonparaxiality, filament formation, and beam breakup in the self-focusing of optical beams, J. Opt. Soc. Am. B 5, 633 (1988).

[33] Jos M. Bioucas-Dias and Gonalo Valadao, Phase unwrapping via graph cuts, IEEE Trans. Image Process. 16, 698 (2007). 